\title{
Solar wind fluctuations and solar activity long-term swing: 1963-2012
}

\author{
Zerbo, J. L. ${ }^{1,2}$, Amory-Mazaudier, C. ${ }^{2}$ and Ouattara, F. ${ }^{3}$ \\ ${ }^{1}$ UFR/ST, Université Polytechnique de Bobo Dioulasso, Burkina Faso \\ ${ }^{2}$ LPP-Laboratoire de Physique des Plasmas/UPMC/Polytechnique/CNRS, \\ UMR 7648, 4 Avenue de Neptune 94107 Saint-Maur-des-Fossés, France \\ ${ }^{3}$ Université de Koudougou, BP 376, Koudougou, Burkina Faso \\ email: jeanlouis.zerbo@gmail.com
}

\begin{abstract}
In this study we investigate the time variation of several solar activity, geomagnetic indices, and solar wind parameters (B, V). It is well known that solar wind is one of the main contributing factors to geomagnetic activity and his topology is strongly affect by solar events such as CMEs and coronals. For these two solar events, we study the correlation between PCI and BV during solar cycle phases and point out the close link between PCI and the occurring of CMEs and high wind speed flowing from coronal holes.
\end{abstract}

Keywords. solar wind, solar activity indices-Joule heating, IMF reversal, PCI

\section{Introduction}

The Sun interacts with the Earth environment throughout radiation and solar wind. This permanent and variable interaction can cause sometimes severe damages to our society. Therefore the Sun-Earth study is so important for general science and the life on Earth as well. Since it is well known that the CMEs occur in majority on the maximum phase of the solar cycle and the high stream solar wind following from coronal holes occur during the declining of solar cycle, we choose in this paper to investigate the natural links between solar wind parameters (B, V) and the Polar Cap Index (PCI) introduced by Troshichev et al., 1988, and used in a statistical sense as a measure of electric field. Our interest is to learn more about the link between the solar dynamo and the energy dissipated in interplanetary medium in the context of geomagnetic activity (Legrand and Simon, 1989; Zerbo et al., 2012).

\section{Data presentation}

Based on the temporal distribution of solar events (CMES, coronal holes) and the fact that he Polar Cap Index can be used as a proxy of Joule heating effect (Francis K. Chun et al., 1999), we investigate the level of the PCI during solar activity. The Figure presented in this work shows the time variations of the Interplanetary Magnetic Field (B), the sunspot number ( Rz), the solar wind speed (V) and the Polar Cap Index during the period 1975-2012. We can remark that the peaks in PCI profile occur near the sunspot maximum and more frequently on the declining of solar cycle. We can assume that the peaks observed in PC index are closely linked with rapid solar wind streams from the coronal holes and CMEs. Using these observations, we investigate the correlation between the PCI and the BV and find the most interesting results, about $\approx 70 \%$., on the maximum and declining phase of solar cycle. These results show that PCI is closely 

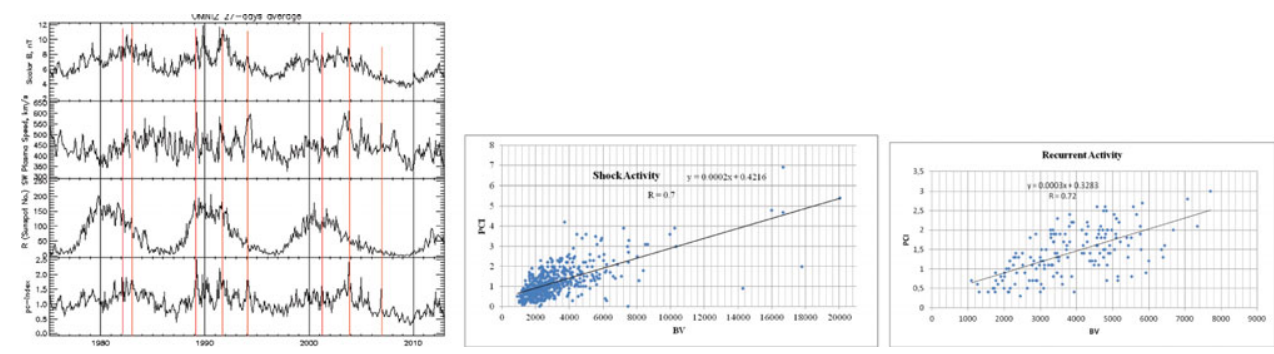

Figure 1. left panel: Time profiles of the Interplanetary Magnetic Field (B), the sunspot number $(\mathrm{Rz})$, the solar wind speed (V) and the Polar Cap Index during the period 1975-2012, middle panel: Correlation between PCI and BV for Shock activity, right panel: Correlation between PCI and BV for Recurrent activity.

linked to BV and inform at the same time on the effects of solar wind and CMEs in the interplanetary medium and terrestrial atmosphere during the two solar cycle phases when the most important energy (Shock and high stream solar wind, Legrand and Simon, 1989 ) is bring on the interplanetary medium by solar radiation.

\section{Conclusion}

The important remark is that the highest energy is produced near the sunspot maximum and especially during the declining phase of solar cycle. This study shows that to investigate the interconnection between solar activity indices is very important for forecasting the solar activity and to learn how the terrestrial atmosphere reacts during solar events. Our results point out the necessity to do studies for each class of solar events in order to know the different characteristics of the coupling between solar wind and magnetosphere.

\section{References}

Francis, K., et al. 1999, Polar cap index as a proxy for hemispheric Joule heating, J. Geophys. Letter, vol. 26, NO. 8, p. 1101-1104.

Legrand, J. P. \& P. A. Simon 1989, Solar cycle and geomagnetic activity: A review for geophysicists. Part I. The contributions to geomagnetic activity of shock waves and of the solar wind. Annales geophysicae, $7,(6)$.

Ouattara, F. \& C. Amory-Mazaudier 2009, Solar geomagnetic activity and Aa indices toward a Standard, J. Atmos. Solar-Terr. Phys, 71, pp. 1736-1748.

Troshichev, O. A., V. G. Andrezen, S. Vennerstrm, \& E. Friis-Christensen 1988, Magnetic activity in the polar cap A new index, Planet. Space Sci., 36, 1095.

Zerbo, J.L., Amory-Mazaudier, C., Ouattara, F., \& Richardson, J. 2012, Solar wind and geomagnetism, toward a standard classification 18682009. Ann Geophys. 30, 4216. 